\title{
Solubilization Process
}

\section{solubilization}

\section{Three Stage Model}

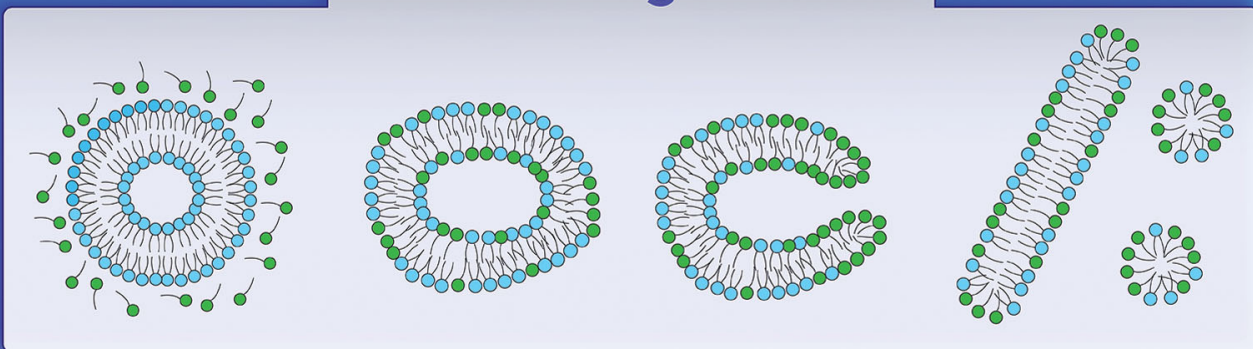

Showcasing research from the group of $\mathrm{Dr}$ Giuseppe Milano, Department of Chemistry and Biology "A. Zambelli",

University of Salerno, Salerno, Italy

Biomembrane solubilization mechanism by Triton X-100: a computational study of the three stage model

The solubilization of a lipid membrane by Triton X-100 is investigated at molecular resolution using molecular dynamics simulations. Thanks to large time and length scales accessible by the hybrid particle-field approach, it is possible to monitor the whole process, and to confirm the three stage model for the solubilization mechanism.

\section{As featured in:}

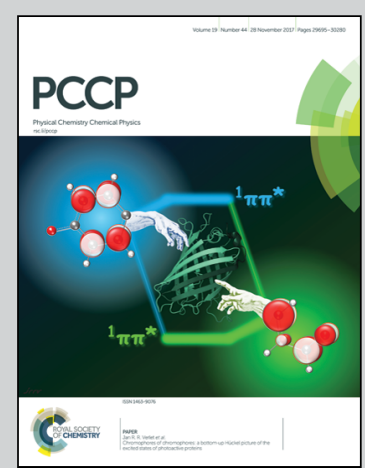

See Antonio De Nicola,

Giuseppe Milano et al.,

Phys. Chem. Chem. Phys., 2017, 19, 29780. 
Check for updates

Cite this: Phys. Chem. Chem. Phys., 2017, 19, 29780

Received 9th June 2017,

Accepted 6th September 2017

DOI: $10.1039 / c 7 c p 03871 b$

rsc.li/pccp

\section{Biomembrane solubilization mechanism by Triton $X-100$ : a computational study of the three stage model $\dagger$}

\author{
Antonio Pizzirusso, ${ }^{a}$ Antonio De Nicola, (D)*a G. J. Agur Sevink, (D) ${ }^{\mathrm{b}}$ Andrea Correa, \\ Michele Cascella, (D) ${ }^{d}$ Toshihiro Kawakatsu, ${ }^{e}$ Mattia Rocco, (D) ${ }^{f}$ Ying Zhao, ${ }^{9}$ \\ Massimo Celino ${ }^{h}$ and Giuseppe Milano*a
}

\begin{abstract}
The solubilization mechanism of lipid membranes in the presence of Triton X-100 (TX-100) is investigated at molecular resolution using molecular dynamics (MD) simulations. Thanks to the large time and length scales accessible by the hybrid particle-field formulation of the models employed here, the complex process of membrane solubilization has been studied, with the goal of verifying the three stage model reported in the literature. DPPC lipid bilayers and vesicles have been studied at different concentrations of the TX-100 detergent employing coarse grained (CG) models. Systems up to $\sim 600.000$ beads, corresponding to more than 2 millions heavy atoms, have been simulated. Moreover, in order to clarify several experimental pieces of evidence, both slow and fast detergent partition scenarios have been investigated. Flat and curved (vesicles) lipid bilayer surfaces, interacting with TX-100, have been considered to study the curvature effects on the detergent partition rate in the membrane. Shape and conformational changes of mixed DPPC/TX-100 vesicles, as a function of TX-100 content, have also been studied. In particular, high curvature surfaces, corresponding to a higher local TX-100 content, promote a membrane rupture. In flat lipid surfaces, on the time scale simulated the detergent partition is almost absent, following a different pathway of the solubilization membrane mechanism.
\end{abstract}

\section{Introduction}

Membrane solubilization (MS) by interaction with detergents is a crucial step in several technological applications of critical importance, among which is the characterization of integral membrane proteins. The use of detergents that are able to

${ }^{a}$ Dipartimento di Chimica e Biologia, Università di Salerno, Via Giovanni Paolo II, 132, I-84084, Fisciano, Italy.E-mail: adenicola@unisa.it, gmilano@unisa.it

${ }^{b}$ Leiden Institute of Chemistry, Leiden University, P. O. Box 9502, 2300 RA Leiden, The Netherlands

'Department of Chemical Science, Federico II University of Naples, Via Cinthia, Complesso Monte S. Angelo, 80126 Napoli, Italy

${ }^{d}$ Department of Chemistry and Hylleraas Centre for Quantum Molecular Sciences, University of Oslo, Sem Scelands vei 26, 0371 Oslo, Norway

${ }^{e}$ Department of Physics, Tohoku University, Aoba, Aramaki, Aoba-ku, Sendai 980-8578, Japan

${ }^{f}$ Biopolimeri e Proteomica, Ospedale Policlinico San Martino, Largo R. Benzi 10 I-16132, Genova, Italy

${ }^{g}$ Institute of Nano-Photonics, School of Physics and Materials Engineering, Dalian Minzu University, Dalian 116600, China

${ }^{h}$ ENEA, C.R. Casaccia, Via Anguillarese 301, I-00123 Roma, Italy

$\dagger$ Electronic supplementary information (ESI) available: Composition of simulated systems, PF-CG model parameters, gyration radii calculation, the size of pure DPPC vesicles in water, additional analysis of mixed vesicles. See DOI: 10.1039/c7cp03871b extract proteins from their native membrane and isolate them in vesicles ${ }^{1-5}$ allows the study of macromolecular interactions with other proteins as well as the study of the same systems in protomeric and self-associated states. ${ }^{6}$ Studies using detergents for the isolation of proteins in specific membrane domains have so far allowed important physiological understanding in processes such as surface signaling, ${ }^{7}$ cell adhesion, ${ }^{7-10}$ and intracellular sorting. ${ }^{11-13}$

MS is also crucial in targeted drug delivery processes, where control over liposome solubilization and membrane poration mechanisms constitutes some essential knowledge for the release of the compounds of interest. ${ }^{14}$

As claimed by several authors, ${ }^{1,2,15-21}$ the solubilization of lipid bilayers is a complex mechanism that depends both on the detergent type and concentration, and on the phase state of the lipid membrane ${ }^{16,17}$ Experimentally, MS is characterized by two measurable values of the detergent/lipid molar ratio $(R) .{ }^{15,20}$ The first value is the saturation limit $\left(R_{\mathrm{e}}^{\text {sat }}\right)$ representing the detergent/lipid ratio at which mixed micelles begin to form, still in the presence of a stable lipid bilayer. ${ }^{22-24}$ The second is the solubilization limit $\left(R_{\mathrm{e}}^{\mathrm{sol}}\right)$, at which complete membrane solubilization occurs, and the whole lipid content is found in mixed lipid/detergent aggregates. ${ }^{22-24}$ Both quantities contribute 
to determine the best experimental conditions needed to solubilize a lipid bilayer, and they depend on temperature and on the lipid phase present at a given temperature. ${ }^{22}$ In particular, in ref. 22 it was shown how the solubilization of DPPC is a relatively slow process below the gel to liquid-crystalline transition temperature $\left(T_{\mathrm{m}}\right)$. In the gel phase, in fact, the detergent function of TX-100 is reduced and the TX-100 concentration required for solubilizing DPPC decreases with temperature up to $T_{\mathrm{m}}$, where the liquidcrystalline phase transition occurs. However, at temperature values higher than $296 \mathrm{~K}, R_{\mathrm{e}}^{\mathrm{sat}}$ and $R_{\mathrm{e}}^{\mathrm{sol}}$ values become comparable with those of the fluid phase. ${ }^{22}$

Thus, the TX-100/DPPC ratio is not the only parameter that controls the solubilization process. TX-100 interacts with DPPC bilayers also depending on the physical state of the lipid (gel or fluid) which in turn depends on the temperature. ${ }^{22,25}$ In particular, the gel-fluid transition temperature $\left(T_{\mathrm{m}}\right)$ is also influenced by the TX-100 concentration; in fact, according to Goni and co-workers, ${ }^{21}$ increasing the amount of TX-100 the DPPC $T_{\mathrm{m}}$ decreases by a few degrees. In particular, Goni's work can be considered as the pioneering study on the mechanism of biological membrane solubilization by some detergents, still playing a prominent role in this field and providing important information. Since the late eighties, complex processes such as the solubilization of sonicated unilamellar vesicles have been investigated with turbidity measurements. ${ }^{26}$ Although several studies show, as expected, evidence of fast solubilization of TX-100 in the lipid phase, ${ }^{3,27-32}$ some show solubilization of TX-100 also in the gel phase of different phosphatidylcholines. In some cases, the solubilization in the gel state required less detergents than in the liquid-crystalline one. ${ }^{25,33}$

Triton X-100 is one of the most studied detergents in the field of membrane solubilization, and it is largely used as a reference for comparing the solubilization activity of other detergents, as recently reported in ref. 34, where the effects of the surfactant electric charge and of its alkyl chain length on the solubilization process have been tested.

Different phosphotidylcholine solubilization states in the form of large unilammellar vesicles have also been investigated by TX-100 as a surfactant and grouped according to their chemical properties, ${ }^{35}$ finding a moderate decrease in the TX-100/lipid solubilization ratio as the chain length increases in saturated phospholipids, or an increment of this ratio when double bonds are introduced, especially for cis-isomers.

Recent studies of Heerklotz and co-workers, who employed and developed isothermal titration calorimetry (ITC) to investigate the interaction of surfactants with membranes, deserve also to be mentioned. ${ }^{31,32,36-39}$ With this technique they were able to study in more detail the TX-100 insertion in the bilayer membrane, a complex process consisting in the detergent partitioning and micelle formation, often accompanied by a heat consumption or release. In particular, the detergent partitioning in the lipid membrane can be quantitatively measured with the partition coefficients, in turn correlated with the corresponding critical micellar concentration, as reported in ref. 31 and 36 in which Triton is allocated as the strong detergent. TX-100 loses its detergent function in the presence of sphingolipids and cholesterol in unsaturated phosphatidylcholine, and several papers $^{3,29,40}$ suggest the hypothesis of lipid rafts consisting of liquid ordered phases where TX-100 fails to insert. Using the ITC technique, Heerklotz and co-workers have also investigated this hypothesis observing the coexistence of two separate domains, an ordered detergent-resistant membrane and a fluid TX-100-rich domain. ${ }^{32,38}$ A similar behavior can be observed for pure phosphatidylcholine at very low temperature (at about $4{ }^{\circ} \mathrm{C}$ ), when the lipids are in the gel phase..$^{3,22}$

The insolubility by Triton in the presence of sphingolipids and cholesterol is a hotly debated topic in the literature. For instance Lichtenberg, Goni and Heerklotz specified how the concepts of the detergent-resistant membranes (DRMs), lipid rafts, and liquid ordered phase are often confused with one single process. While DRMs are the result of an incomplete solubilization of membranes by a detergent, rafts consist in membrane domains enriched in sphingolipids and cholesterol, completely independent of the use of the surfactant, although the Triton-insolubility is due to the liquid-ordered phase of these domains. ${ }^{41}$

However, the addition of TX-100 promotes the formation of liquid-ordered domains ${ }^{37}$ as a result of an unfavourable interaction between TX-100 and an order-preferring lipid such as sphingolipids, ${ }^{39}$ or between TX-100 and cholesterol. ${ }^{38}$ In these cases, TX-100 can act as a heterogeneous perturbation agent, when the interaction is favourable in the presence of disorderpreferring lipids, such as phosphatidylcholines or POPC, or it can act as a homogeneous perturbation agent, directly solubilizing the membrane. ${ }^{38}$

One of the most accepted mechanisms to describe the solubilization process is the three-stage model proposed by Helenius and Simons. ${ }^{1}$ This model can be ideally divided into the following steps: (i) initial interactions between the detergent and lipids that do not yield micellar structures; only a detergent partition in the lipid bilayer occurs until saturation is reached. (ii) Perturbation of the bilayer structure induced by the detergent, producing the conversion of the detergent saturated bilayer into mixed micelles. (iii) Size reduction and dispersion into the solvent of the mixed micelles, as a result of their interaction with more detergent added to the solution.

The detergent partition between lipid layers is commonly considered to be rapid, but such an assumption does not hold for all cases. In fact, as observed by Stuart et al. ${ }^{42}$ and KraghHansen et al., ${ }^{43}$ on the basis of cryo-transmission electron microscopy (Cryo-TEM) and turbidimetry experimental measurements, the detergent partition appears to be a more complex process. In particular, based on fast or slow detergent trans-membrane swapping times (a motion commonly referred to as flip-flop), Lichtenberg ${ }^{16,17,42,43}$ proposed two possible modifications to the original mechanism. In the first case, the fast detergent flipping between layers leads to a solubilization process via open vesicular intermediates, ${ }^{42}$ or a trans-bilayer attack. ${ }^{44}$ In contrast, in the slow detergent ${ }^{42,43}$ flip-flop limit, the system goes through a non-equilibrium state caused by detergent mass imbalance in the outer lipid layer. In this case, the solubilization mechanism can occur via the binding of detergent 


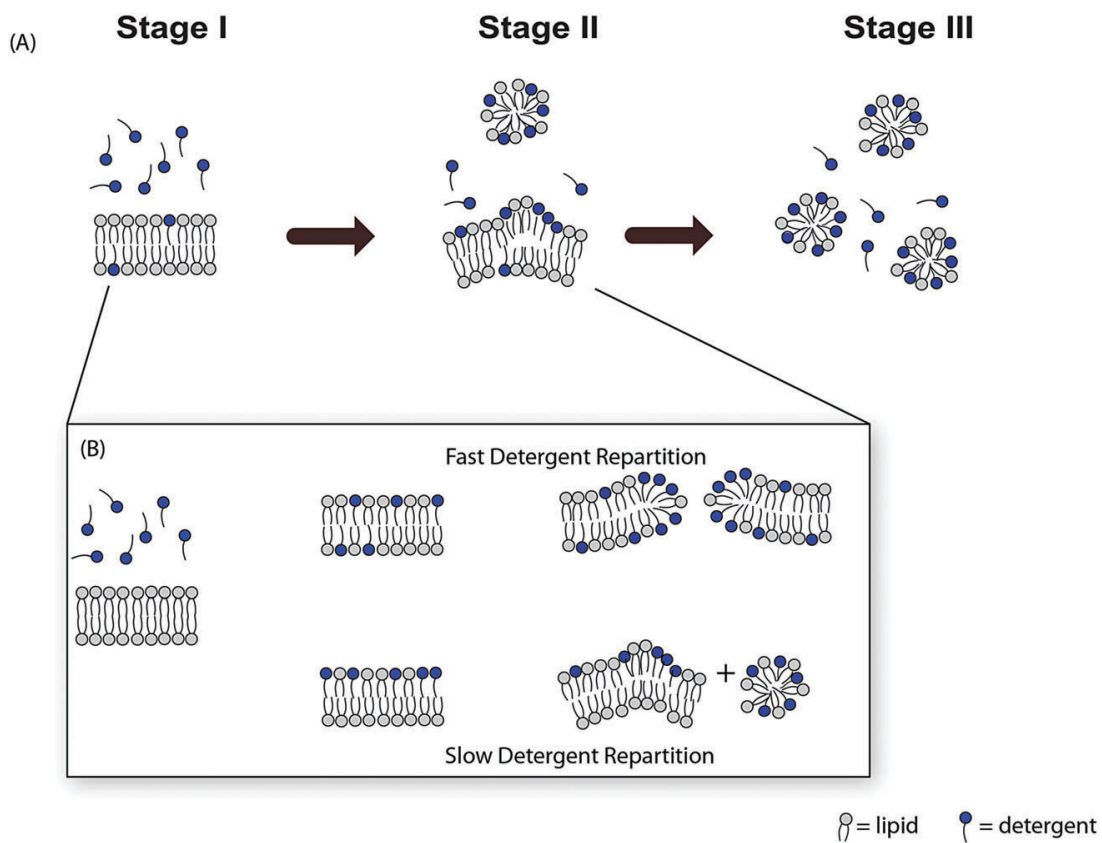

Scheme 1 (A) Illustration of the three stage model. ${ }^{1}$ The detergent molecules approach a lipid layer (stage I). Formation of mixed lipid/detergent aggregates (stage II). The whole lipid bilayer is converted into mixed micelles (stage III). (B) Solubilization pathways of slow and fast detergent partition (flip-flop).

micelles to the bilayer with an extraction of lipids from them, ${ }^{42}$ or with the formation of mixed lipid/detergent aggregates, as suggested by Goni (Scheme 1). ${ }^{16,17,45}$

Ambiguities left on the molecular mechanisms associated with MS are due to the lack of experimental methods able to characterize the whole process. For example, Cryo-TEM or light scattering $^{46,47}$ can study the shape and size of liposomes and mixed detergent/lipid micelles on large size length scales. Nonetheless, neither of these techniques can ensure reliable results when small and large aggregates are present in solution at the same time. In contrast, ${ }^{31} \mathrm{P}-\mathrm{NMR}$ spectroscopy yields accurate narrow signals for small particles, ${ }^{27,48,49}$ but peak broadening in large aggregates leads to signal loss below detection. Thermodynamic data can be properly measured during the solubilization process using ITC, ${ }^{50,51}$ but it is not possible to have any information about the size and the shape of the aggregates formed during the process. Finally, turbidimetry measures the concentration of the detergent needed to solubilize the lipid membrane, ${ }^{25,52,53}$ monitoring the loss of transmitted light due to the scattering of aggregates present in solution, but this method does not yield accurate information about the shape and composition of mixed aggregates.

Computer modelling provides an effective way of characterizing the molecular details of several mechanisms. In the past, computational studies were successfully applied to investigations on lipid vesicle fusion, ${ }^{54}$ pore formation in lipid bilayers, ${ }^{55}$ selfassembly of lipid membrane-protein, ${ }^{56,57}$ flip-flop of lipids in biomembranes, ${ }^{58}$ cholesterol flip-flop in lipid bilayers, ${ }^{59}$ curvature effects on lipid packing in liposomes, ${ }^{60}$ depletion of cholesterol from membranes mediated by cyclodextrin, ${ }^{61}$ and transition of lipid vesicles to tubular networks mediated by proteins. ${ }^{62}$ The cited examples are similar to the mechanisms involved at different stages of the MS process. For these reasons, computer modelling can provide the characterization, at the molecular level, of a complex mechanism such as MS.

In this work, we studied the solubilization process of phospholipid bilayers at the molecular level of detail using molecular dynamics (MD) simulations. ${ }^{63}$ In particular, we adopted a molecular coarse-grained (CG) resolution, which provides the best compromise between access to longer time-scales and retaining of molecular-level details with respect to more expensive atomistic simulations. ${ }^{64}$ CG models were successfully applied to the study of a variety of collective phenomena involving soft-matter systems, including biological membranes, ${ }^{64-73}$ detergents $^{40,65,74-80}$ and protein systems. ${ }^{57,81}$ Examples of studies about lipid membranes interacting with surfactants have been reported by several authors. ${ }^{40,65,74,79,80}$ In particular, Muddana et al. ${ }^{40}$ studied the phase separation of lipid bilayers due to the presence of amphiphilic molecules such as Triton, benzyl alcohol and vitamin E. Pizzirusso et al. ${ }^{74}$ described the effect of the TX-100 content on the area/molecule ratio in dipalmitoylphosphatidylcoline (DPPC) mono- and bilayers. Groot and Rabon $^{79}$ reported a study of phosphatidylethanolamine membranes in the presence of the hexaethylene glycol monododecyl ether $\left(\mathrm{C}_{12} \mathrm{E}_{6}\right)$ surfactant at different molar ratios, using Dissipative Particle Dynamics (DPD). They found that starting from a content of $30 \%$ of $\mathrm{C}_{12} \mathrm{E}_{6}$, lipid membranes form holes, while at a higher surfactant content they observe the membrane rupture. Lin et al. ${ }^{80}$ studied, with the same DPD technique, the effect of hydrophobicity of a nonspecific surfactant model in the solubilization process of vesicles. In particular, they found that only surfactants with suitable hydrophobicity are able to solubilize a vesicle. Moreover, Bandyopadhyay 
et $a .^{65}$ have studied the effect of anionic surfactants on the orientation and order of lipids and on the localization of surfactants in membranes.

To extend even further the simulation times and length scales required to observe slow collective events such as membrane solubilization (not easily accessible by standard MD methods even using CG models), we coupled CG molecular representations to an innovative MD technique, based on a hybrid particle-field representation $^{63,82}$ called Molecular Dynamics-Self Consistent Field (MD-SCF). MD-SCF has already been successfully applied to study the phase behaviour of phospholipid/water mixtures, ${ }^{73,83}$ Pluronics block-copolymer/water mixtures, ${ }^{84}$ TX-100/water mixtures, ${ }^{75}$ and Pluronic surfactant aggregates interacting with lipid bilayers. ${ }^{85}$ This technique is becoming popular and is aimed toward an accurate description of morphological changes of biomembranes using models at chemical resolution. ${ }^{86}$

As a test case, we investigated the solubilization of DPPC bilayers by TX-100. TX-100 was chosen because it is one of the most employed non-ionic detergents in applications of biological interest, such as solubilization of lipid bilayers, ${ }^{30}$ or isolation and purification of membrane proteins. ${ }^{5,20}$

Our study covers the solubilization properties at DPPC/ TX-100 ratios both lower and higher than the experimental saturation and solubilization molar ratio $^{22}\left(R_{\mathrm{e}}^{\mathrm{sat}}\right.$ and $\left.R_{\mathrm{e}}^{\mathrm{sol}}\right)$ limits. It is worth noting that these limits are affected both by the temperature and the lipid phase in which the bilayer persists, ${ }^{22}$ and from this point of view we estimate the solubilization process with different DPPC/TX-100 ratios in a range of values lower and higher than the experimentally obtained $R_{\mathrm{e}}^{\mathrm{sat}}$ and $R_{\mathrm{e}}^{\mathrm{sol}}$ corresponding to a given temperature.

The present study is organized as follows. A brief description of the hybrid MD-SCF approach, together with the description of CG models and simulation details are presented in Section 2. The results and discussion, Section 3, are divided into three parts: first the validation of CG models is shown; subsequently the description of the results about the slow MS mechanism is presented, and then the fast MS mechanism is discussed. Finally, in Section 4 the conclusions are summarized.

\section{MD-SCF approach, CG models and simulation details}

\subsection{MD-SCF approach}

The main feature of the hybrid MD-SCF approach is that the calculation of intermolecular non-bonded forces and potentials, representing the most computationally demanding part of MD simulations, can be substituted by a calculation of external potentials dependent on the local density (at position r). ${ }^{63}$ According to the SCF theory, a multi-body problem, such as molecular motion, can be reduced to a problem of deriving the partition function of a single molecule in an external potential $V(\mathbf{r})$ and to obtain a convenient expression of $V(\mathbf{r})$ and its derivatives.

In the framework of the SCF theory, a molecule is regarded to be interacting with the surrounding molecules through a mean field, rather than direct interactions among the molecules.
It is assumed that the density dependent interaction potential $W$, where each species is specified by the index $K$, takes the form:

$$
\begin{aligned}
& W\left[\left\{\phi_{K}(\mathbf{r})\right\}\right] \\
& =\int \mathrm{d} \mathbf{r}\left(\frac{k_{\mathrm{B}} T}{2} \sum_{K K^{\prime}} \chi_{K K^{\prime}} \phi_{K}(\mathbf{r}) \phi_{K^{\prime}}(\mathbf{r})+\frac{1}{2 \kappa}\left(\sum_{K} \phi_{K}(\mathbf{r})-\phi_{0}\right)^{2}\right)
\end{aligned}
$$

where $\phi_{K}(\mathbf{r})$ is the coarse-grained density of species $K$ at position $r$ and $\chi_{K K^{\prime}}$ are the mean field parameters for the interaction of a particle of type $K$ with the density fields due to particles of type $K^{\prime}$. The second term of the integrand on the right-hand side of eqn (1) is the relaxed incompressibility condition. $\kappa$ is the compressibility that it assumed to be sufficiently small, and $\phi_{0}$ is the total number density of the segments. ${ }^{87}$ It can be shown using the so-called saddle point approximation that the external potential is given by:

$$
\begin{aligned}
V_{K}(\mathbf{r}) & =\frac{\delta W\left[\phi_{K}(\mathbf{r})\right]}{\delta \phi_{K}(\mathbf{r})} \\
& =k_{\mathrm{B}} T \sum_{K^{\prime}} \chi_{K K^{\prime}} \phi_{K^{\prime}}(\mathbf{r})+\frac{1}{\kappa}\left(\sum_{K} \phi_{K}(\mathbf{r})-\phi_{0}\right)
\end{aligned}
$$

To connect the particle and field models for the proposed hybrid MD-SCF scheme, it is necessary to obtain a smooth coarsegrained density function directly from the particle positions. Such a function can be obtained using a mesh-based approach, which must also be able to give the density derivatives required to calculate the forces acting on molecules. The derivation of eqn (2) and the implementation of this approach are reported elsewhere. ${ }^{63,82}$ Details concerning the OCCAM code, used to perform the PF simulations, can be found in ref. 87.

\subsection{Model details}

As the reference particle-particle (PP) simulations, we used CG models based on the Martini force-field ${ }^{67}$ for both phospholipid DPPC ${ }^{67}$ and TX-100. ${ }^{40,74}$ These models have been chosen because the important structural properties, such as the density profile and the area/molecule ratio, were found to match the experimental behaviour. In particular, in a recent work of Pizzirusso and co-workers ${ }^{74}$ the Martini-based CG model of TX-100 has been validated to reproduce the experimental area/ molecule ratio in mixed TX-100/DPPC mono- and bi-layers. The model details are reported in the ESI. $\dagger$

For the hybrid particle-field (PF) simulations, in previous works we had developed phospholipid CG models capable of correctly reproducing, in good agreement with respect to more detailed models and experiments, the main features of bilayers, such as the bilayer thickness and electron density profiles. ${ }^{73}$ Moreover, we also extended and validated such models in the reproduction of morphologies of a water/lipid mixture in nonlamellar phases. ${ }^{83}$ Recently, we have developed a PF CG model of TX-100 able to correctly reproduce the spontaneous selfaggregation of micellar assemblies, aggregation numbers, polydispersity of micelle aggregates, the size and shape of micelles and the dynamic properties of a TX-100/water mixture. ${ }^{75}$ More details of 
PF CG models are reported in the ESI. $\dagger$ Data presented in the remaining of the result sections refer therefore to $\mathrm{PF}$ models only.

\subsection{Computational details}

All PF simulations reported in the present paper have been performed using the parallel version of the OCCAM MD code. ${ }^{87}$ The reference PP simulations were performed with version 4.5.4 of the GROMACS code. ${ }^{88}$ The NPT ensemble was employed for all PP simulations, keeping the temperature constant at $298 \mathrm{~K}$ using a Berendsen thermostat ${ }^{89}\left(\tau_{\mathrm{T}}=1.5 \mathrm{ps}\right)$. A semi-isotropic pressure coupling was used for the NPT ensemble. In particular, a lateral pressure of 1 bar was coupled separately from the normal component pressure ( 1 bar), with respect to the bilayer plane. The coupling constant for the Berendsen barostat ${ }^{89}$ was set to $\tau_{\mathrm{T}}=1.5 \mathrm{ps}$. The non-bonded interactions are modelled by the Lennard-Jones (LJ) pair potential and electrostatic Coulombic potential. For both, a cutoff $r_{\text {cut }}=1.2 \mathrm{~nm}$ was used. The LJ potential was smoothly shifted to zero from the distance $r_{\text {shift }}=0.9 \mathrm{~nm}$ to the cutoff. The neighbour list was updated every 10 steps. A time step of 0.03 ps was employed for all PP and PF simulations. The PF simulations were performed in the NVT ensemble, and the temperature was controlled, at $298 \mathrm{~K}$, by using the Andersen thermostat ${ }^{90}$ with a collision frequency of $7 \mathrm{ps}^{-1}$. The grid size was set to $0.7 \mathrm{~nm}$, and the density was updated every 300 steps.

We chose to build initial systems by considering two aspects: first, the TX-100/DPPC ratio $R$ and, second, the curvature of the lipid bilayer. According to the experimental $R_{\mathrm{e}}^{\text {sat }}$ and $R_{\mathrm{e}}^{\text {sol }}$ limits obtained $^{22}$ at $T=299 \mathrm{~K}$, we selected three different $R$ values: below $(R=0.1)$, close to the saturation limit $(R=0.43)$, and equal to the solubilization limit $(R=1.0)$. Additional information regarding the construction of the systems and the validation of the interaction between the TX-100 surfactant and a DPPC bilayer in reference to the PP simulations are reported in the ESI. $\dagger$

\section{Results and discussion}

\subsection{Fast-slow detergent partition}

We performed $\sim 5 \mu$ s long simulations for various TX-100/ DPPC systems using molar ratio values either lower or equal to the saturation limit. Different boundary conditions for DPPC were employed. The multiple starting states were chosen with the scope of determining the conditions at which the detergent partition rates fall in either fast or slow regimes.

The DPPC bilayer was organized in two starting states - a flat periodic bilayer, or isolated spherical vesicles of curvature radii $\rho=5.6 \mathrm{~nm}$ and $13 \mathrm{~nm}$. For both DPPC states, different initial distributions of TX-100, at an equal global molar ratio $R$, were prepared. Specifically, TX-100 was either symmetrically distributed in the two DPPC leaflets in equal ratios, or one of the two layers was arbitrarily enriched in TX-100, thus producing an initial asymmetric distribution of the detergent. Therefore, in the symmetric case the effective molar ratio $R_{\mathrm{e}}$ in the two DPPC leaflets was equal to the nominal value $R$, while in the

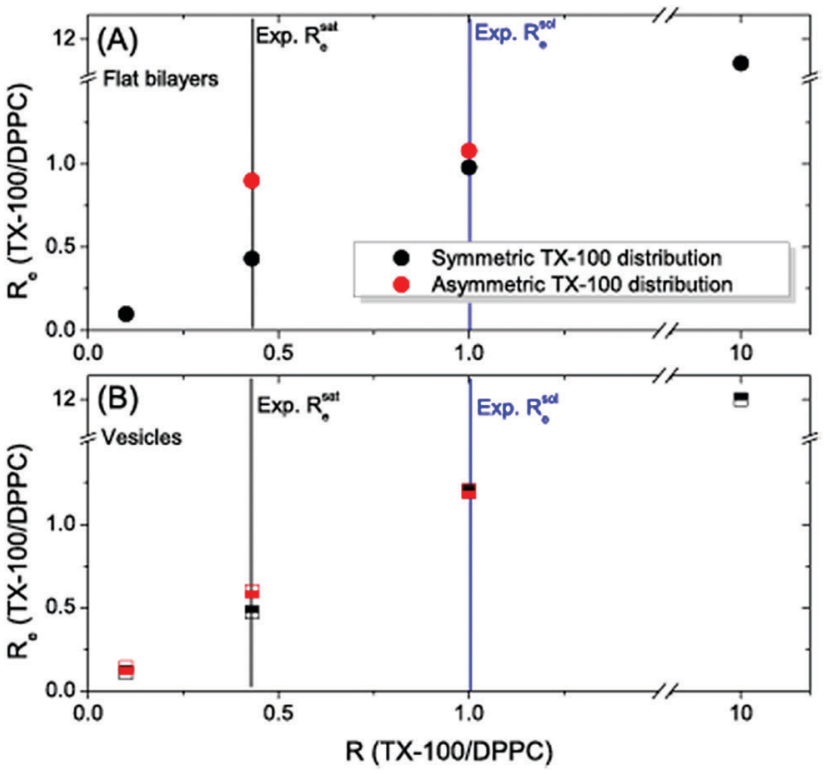

Fig. 1 Effective TX-100/DPPC ratio for: (A) mixed bilayers and (B) mixed vesicles. The black symbols indicate a symmetric random partition of TX-100 between the two layers, while red symbols indicate an asymmetric partition of TX-100. All possible combinations for the TX-100 distributions into bilayers are reported in Table S1 in the ESI. $\dagger$ In the plot, the saturation and solubilization experimental limits for the TX-100/DPPC system at $T=299 \mathrm{~K}$ are also reported. ${ }^{22}$

asymmetric distributions $R_{\mathrm{e}}$ was approximately equal to $2 R$ in the TX-100-enriched layer.

Fig. 1 reports the initial and equilibrium values of $R_{\mathrm{e}}$ after $\sim 4 \mu$ s of simulations for all studied systems (PF systems from VIII to XXVII reported in Table S1 of the ESI $\dagger$ ).

Our data show that, in flat DPPC bilayers, the distribution of TX-100 did not change significantly from the initial values within the simulated time, even if the simulations began from a configuration supposedly far from its thermodynamic equilibrium (for example, from large asymmetric concentrations of TX-100, Fig. 1A). In contrast, for bilayers with a finite curvature radius we observed a fast partition of TX-100 between the two DPPC layers (Fig. 1B), with equilibrium values of $R_{\mathrm{e}}$ very different from the nominal value of $R$. Most importantly, in curved bilayers we observed a constant dynamic exchange of detergent molecules between the leaflets after reaching an equilibrium distribution of the detergent, with an average detergent exchange rate of $\sim 2$ flips per $\mu$ s per molecule. The exchange rate is apparently not affected by the TX-100 concentration, as we found very similar values for increasing ratios $R$ up to the saturation limit. In contrast, in flat bilayers our simulations reported a flipping rate for TX-100 orders of magnitude slower than in curved surfaces $(\sim 0.004$ flips per $\mu$ s per molecule). Our simulations thus show that the radius of curvature of the bilayer is a critical order parameter determining fast or slow partition rates of the detergent.

\subsection{Flat surface bilayers}

Simulations of flat DPPC membranes were run in the presence of TX-100 at three relative concentrations $R=0.43,1.0$, and 10 , 
corresponding to saturation, solubilization and over-solubilization conditions, respectively. In the first two cases $(R=0.43, R=1.0)$, two different initial conditions were considered - one with TX-100 equally distributed between the two layers of the membrane, and one with TX-100 inserted in only one layer. In the third case $(R=10)$ only a symmetric distribution was considered, due to the high molar fraction of TX-100 $\left(\chi_{\mathrm{TX}-100}=0.9\right)$.

Fig. 2 reports key points in the MS mechanism for the five simulated systems as a function of time. As mentioned in the former paragraph, very slow TX-100 partition occurred on the timescale of our simulations $(\sim 5 \mu \mathrm{s})$, independently on the initial distribution of TX-100 with respect to the total amount of TX-100 in the bilayer.

Under the experimental solubilization limit condition $(R=1.0)$ we observed the formation of mixed aggregates of different sizes, and the development of transient holes in the bilayer. Destabilization of the bilayer was in fact achieved already at $R=0.43$ in the presence of an asymmetric distribution of TX-100 in only one leaflet. In this case, we observed the appearance of surface distortions, like budding and invaginations. Due to the slow detergent redistribution, the saturation limit
( $R=0.43)$ corresponds to an effective ratio $R_{\mathrm{e}} \sim 0.86$ in the TX-100 enriched leaflet, thus only slightly lower than the solubilization limit. Our finding is in agreement with experimental observations of Kragh-Hanse, ${ }^{43}$ who reported the formation of mixed micelles from detergent-rich layers at concentrations below the saturation limit.

When TX-100 is added in an asymmetrical way on flat bilayers, it causes disorder on the bilayer surface. This is reasonable considering that the insertion of TX-100 causes an area mismatch between the two leaflets. Consequently, local distortions on this surface can be quickly observed. These are connected both to the area mismatch and to the curvophilic characteristic of the detergent, which induces a curvature on the flat surface in order to solubilize it.

For systems with $R=1.0$ and a symmetric distribution, a stable mixed protrusion combined with the presence and formation of holes has been observed. In the case of an asymmetric distribution, an interconnected and continuous worm-like mixed micelle is formed over the outer layer. Less than $40 \%$ of the original DPPC content remained in a bilayerlike structure. Finally, at initial $R=10.0$, a much higher value
(A)
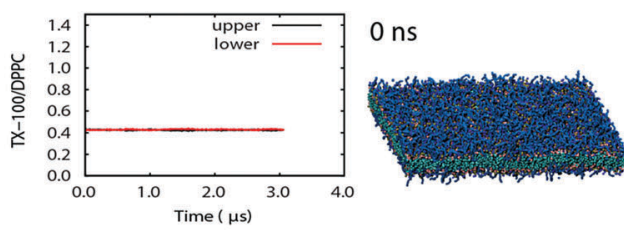

$200 \mathrm{~ns}$

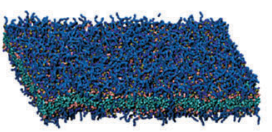

$3.0 \mu \mathrm{s}$

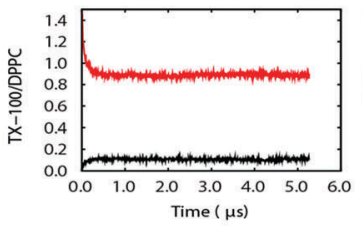

Ons
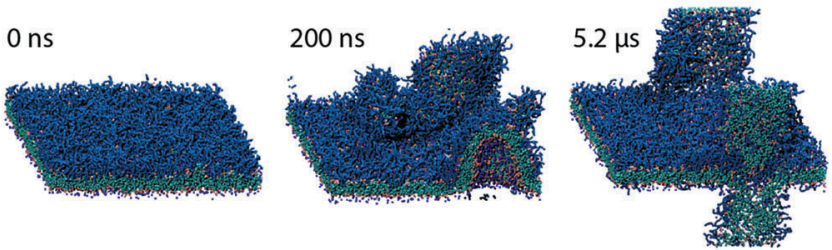

(B)
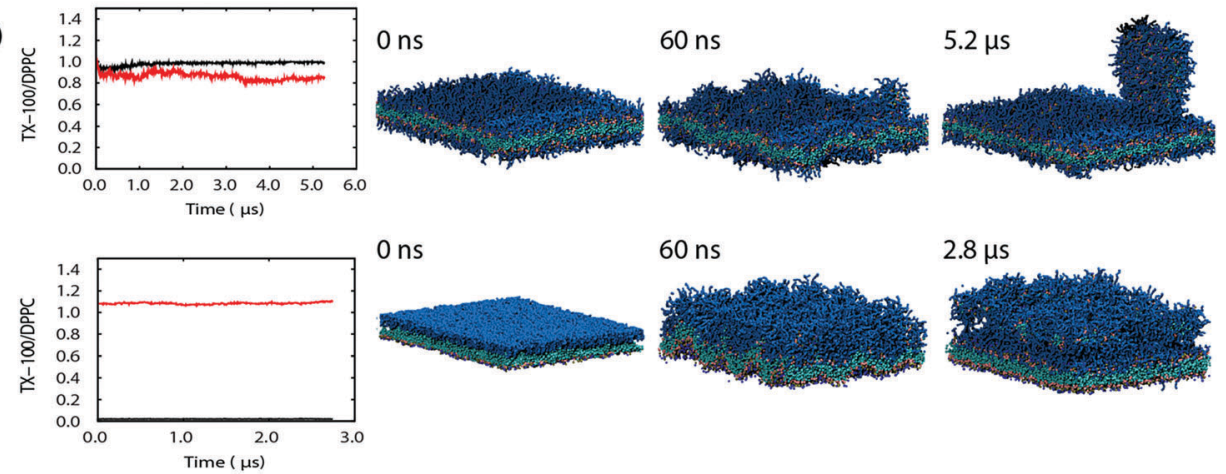

(C)
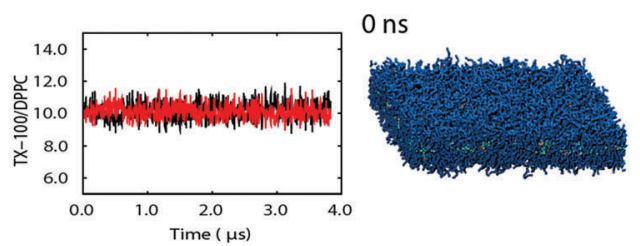

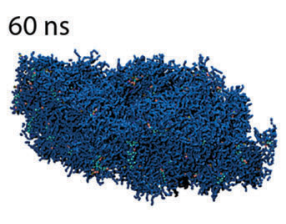

Fig. 2 Time behaviour of the TX-100/DPPC ratio $R$ for: (A) $R=0.43$ for symmetric and asymmetric TX-100 initial distribution. (B) $R=1.0$ and (C) $R=10.0$, only the random initial TX-100 distribution. Next to each plot, snapshots of the flat DPPC bilayer (in grey) with TX-100 molecules (in dark blue) are reported. 

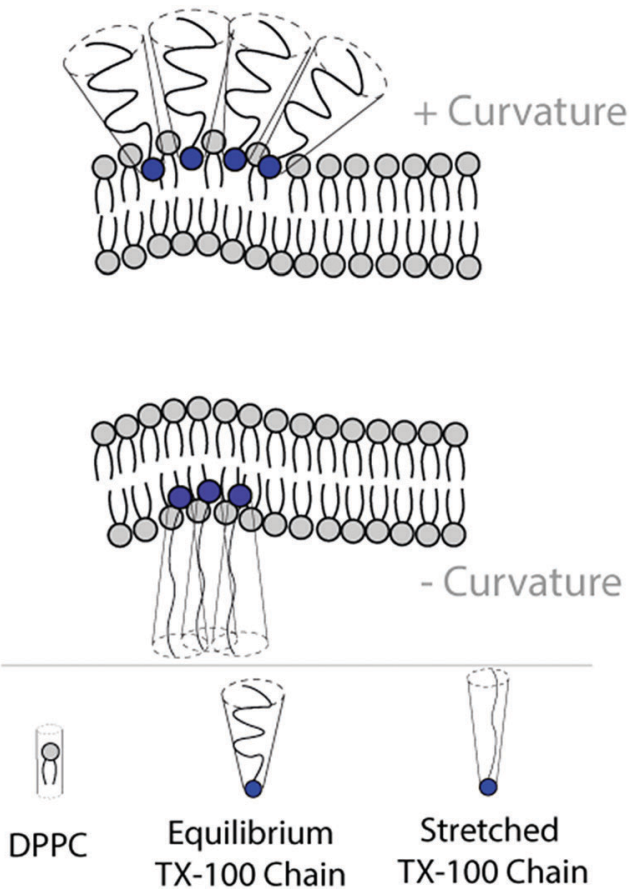

Scheme 2 Scheme representing the packing of TX-100 molecules in a layer with a positive or negative curvature. Together with the scheme are sketched the equilibrium DPPC and TX-100 conformations, and the stretched TX-100 conformation.

than the solubilization limit, we observed a complete disruption of the bilayer in less than $4 \mu \mathrm{s}$, together with formation and interconnection of mixed aggregates (Fig. 2C). Our simulations evidenced that the bilayer distortion events occurring at the solubilization concentration have much faster characteristic times than TX-100 flipping times.

Bilayer deformations induced by TX-100 can be explained taking into consideration the large conformational freedom of the hydrophilic chains of TX-100, which favours the local positive curvature of the bilayer surface, as depicted in Scheme 2. Such an entropy-driven curvophilic behaviour contrasts with the opposite curvophobic behaviour of cylindrical shaped molecules, like phospholipids, which tend to self-assemble along flat surfaces or along surfaces having a small curvature. ${ }^{17}$

\subsection{Curved surface bilayers}

DPPC vesicles with a curvature radius $\rho=5.6 \mathrm{~nm}$ interacting with TX-100 were simulated using four different starting conditions for all three $R=0.1,0.43,1.0$ values. Specifically, TX-100 was (i) randomly distributed into the solvent, simulating the initial dispersion of the detergent into the solution (Fig. 3, state W); (ii) inserted into the outer layer of the DPPC vesicle (state O), simulating initial adsorption from the solvent; (iii) evenly distributed between the inner and outer DPPC, as the hypothetical state where TX-100 has no preference for the two DPPC layers (state S). Finally, a fourth state (iv) where the total amount of TX-100 is distributed in the inner layer of lipid vesicles was considered (state I). Fig. 3 reports TX-100 partition as a function of time for all vesicle systems, and for all the four initial TX-100 distributions.
For systems at low TX-100 concentrations $(R=0.10$ to 0.43$)$, we observed equilibration of the detergent between the two leaflets, occurring in the first $2 \mu$ s of our simulations. The apparent discrepancy in the distribution for the S setup is due to the small size of the systems, corresponding to a difference of 3-5 TX-100 molecules, therefore within the statistical error of the simulations. As expected, we found a preference of TX-100 for the external layer, characterized by the positive curvature. In all cases, starting (I) configurations resulted very far from the thermodynamic equilibrium, and an initial very fast expulsion of TX-100 from the inner leaflet of the bilayer was observed.

At TX-100 concentrations close to the solubilization limit $(R=1.0)$ the DPPC vesicle remained stable only for a short time (between 1 and $2 \mu \mathrm{s}$, according to the starting conditions), and eventually dissolved via different mechanisms like formation of mixed micelles, poration and rupture. The rupture events correspond to accumulation of TX-100 in the inner leaflet to an effective ratio $R_{\mathrm{e}} \sim 0.4$ or more, which corresponds roughly to the nominal saturation concentration.

\subsection{Vesicle poration induced by TX-100}

The flipping of TX-100 between the two DPPC layers requires local molecular reorientation. Specifically, TX-100 molecules need to invert the hydrophobic-hydrophilic portions to migrate between the two layers. Collectively, rearrangement of several TX-100 molecules may promote the formation of transient holes in the structure of the bilayer. Fig. 4 reports the characteristic time of poration for all vesicle systems studied using our simulations. As can be seen from the data reported in Fig. 4, for $R_{\mathrm{e}}$ close or higher than the saturation limit $R_{\mathrm{e}}^{\text {sat }}$, the pores are developed in about $220 \mathrm{~ns}$. Moreover, for ratios $R \geq R_{\mathrm{e}}^{\mathrm{sat}}$ mixed micelle formation and vesicle rupture were observed, in agreement with experimental data. ${ }^{1,16,17,22} \mathrm{We}$ considered the formation of a stable pore whenever there existed a channel that brings into contact the inner and outer regions of the vesicle for long enough to allow solvent diffusion through it. Fig. 4 reports the mean square displacement (MSD) of the water molecules residing inside the vesicle before and after the pore formation (blue line in Fig. 4) for a system with random initial TX-100 distribution at a ratio $R=0.43$. As expected, the MSD is composed of two clear different regimes, corresponding to a neat increase in the diffusion coefficients. In fact, focusing on the water molecules that are inside the cavity formed by a vesicle, we are able to follow their trajectories and quantify their path using the mean square displacements. When the water molecules are forced to stay inside the vesicle their path is confined in a region and the possibility of movement is limited. When the pore instead is formed, these molecules can explore the space outside the vesicle that consists only of bulk water with a consequent increase of the diffusion values. On the basis of these considerations, the water included in the cavity of the vesicle has a slower diffusional regime due to the physical constraints, and therefore this diffusion becomes faster when water molecules are expelled through the formation of a pore. In Fig. 4 the characteristic times needed to develop pores in vesicles 


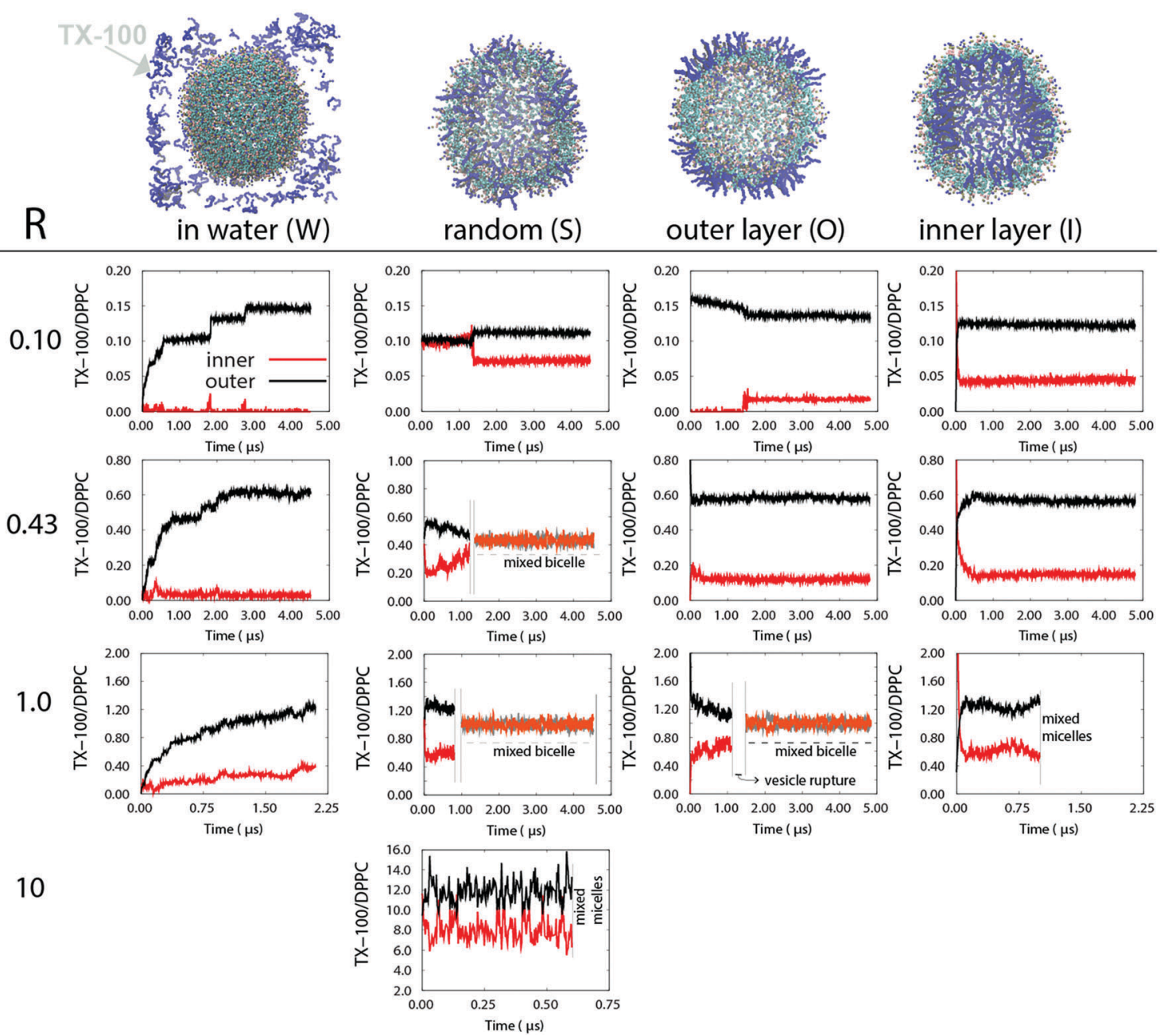

Fig. 3 Time behaviour of different TX-100/DPPC ratios $R$ for vesicles at different initial TX-100 distributions.

with a random TX-100 initial distribution and the time needed to observe complete disruption are also reported as a function of the $R$ ratio.

In Fig. 5, the sequence of events leading to pore formation and rupture of the vesicle for a system with random initial TX-100 distribution at $R=1.0$ are reported. As can be seen, at the starting time the vesicles are perfectly spherical. This sphericity is lost during the simulation time, and it becomes useful to show these snapshots from two different viewpoints, in order to appreciate at the same time the hole formation and the loss of sphericity. After 390 ns holes are completely formed, while at $t=800 \mathrm{~ns}$ only one hole remains stable and the vesicle shape starts to become ellipsoidal. After $4 \mu \mathrm{s}$ the vesicle is completely micellised. From the snapshots, it is evident that the shape of the vesicle changes dramatically during the whole simulation time. During the evolution of the bilayer structure, TX-100 accumulated in high curvature regions. As an example, the snapshots at 390 and 580 ns show configurations in which TX-100 is mainly located on the curved pore edge. Such a behaviour is in agreement with the edge active definition and experimental observation reported by Fromherz. ${ }^{91}$

\subsection{Evolution of DPPC vesicles upon interaction with TX-100}

3.5.1 Effect of the TX-100 concentration on the vesicle shape. We measured the vesicle radius as a function of TX-100 content starting from $R=0$ (pure DPPC vesicle) to $R=1.0$ for a system with the initial random TX-100 distribution (Fig. 6). In agreement with experimental observations, ${ }^{4,28,92-94}$ we found a roughly linear radius increase with increasing content of TX-100 (see the ESI $\dagger$ for data on vesicle radii as a function of time ad different $R_{\mathrm{S}}$ ).

Quantitative analysis of the variations in the vesicle shape as a function of TX-100 concentration was performed by estimating the statistical distributions of the ellipsoids' principal semi-axis lengths $(a, b, c)$ of the simulated vesicles. In the particular case of $a=b=c$, we have a spherical shape. For $a>$ $b=c$, where $a$ is the longest semi-axis, the shape is defined as the prolate, while for $a=b>c$ the shape is defined as the oblate. 


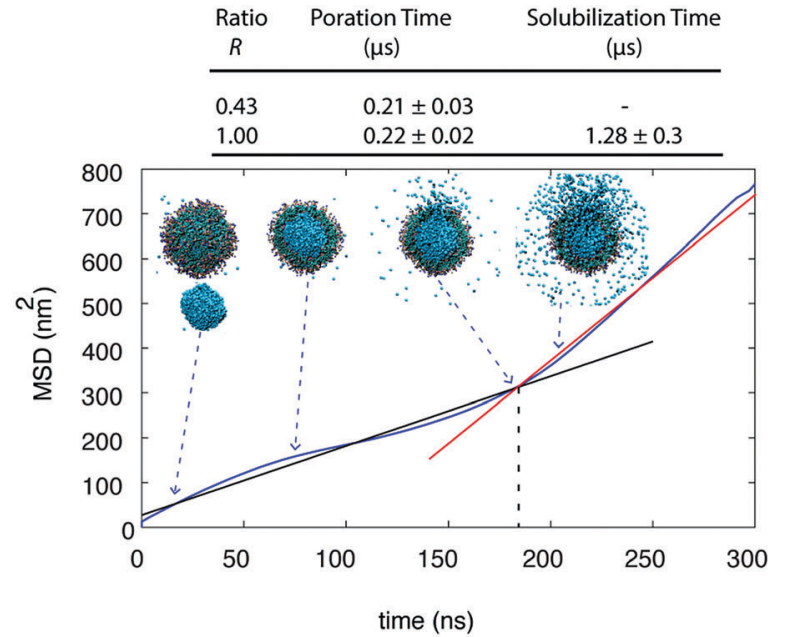

Fig. 4 Top table: Characteristic times needed to develop pores in a vesicle and time to observe complete disruption are reported as a function of the TX-100/DPPC ratio $R$ for systems of the initial random TX-100 distribution. Bottom plot: Mean square deviation (MSD) of the water molecules inside the vesicle cavity (blue curve) of a system with random initial TX-100 distribution at ratio $R=0.43$. The black and red lines represent the linear fittings of different MSD trends, before and after the pore formation. Representative snapshots of vesicles (only lipids) and water molecules inside the vesicle cavity (light blue beads) at different times are shown in the upper part of the plot, describing the entire pore formation process, and the water molecules exiting from the cavity.
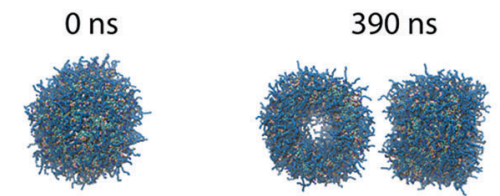

$800 \mathrm{~ns}$

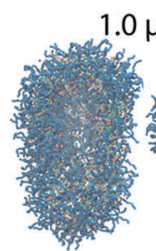

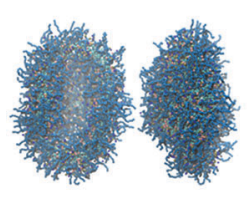

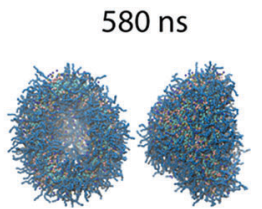

$4.0 \mu \mathrm{s}$

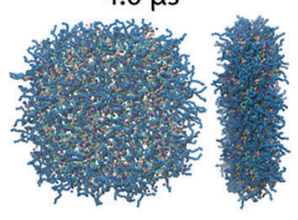

Fig. 5 Snapshots taken during the whole trajectory of the system with the initial random TX-100 distribution at $R=1.0$, showing the vesicle disruption process. The TX-100 molecules are shown in dark blue. The lipid molecules are shown in grey (aliphatic chains) and light green (polar heads).

Fig. 7A reports the statistical occurrence of spherical, prolate or oblate vesicles, as a function of the relative TX-100 concentration. Pure DPPC vesicles have stable spherical shapes, as expected. For a low TX-100 content our simulations predicted the concomitant presence of spherical and slightly ellipsoidal shapes (the latter equally distributed between the oblate and prolate). For $R$ higher than the saturation limit, by strictly analysing the structures of intact vesicles our simulations evidenced mainly prolate shapes, even if the presence of oblate vesicles remained statistically relevant.

In Fig. 7B, the distributions of the shape distortion grade (asphericity), calculated as the ratio between longest $\left(\boldsymbol{r}_{\max }\right)$ and shortest $\left(\boldsymbol{r}_{\text {min }}\right)$ semi-axes, are reported. A low TX-100 content

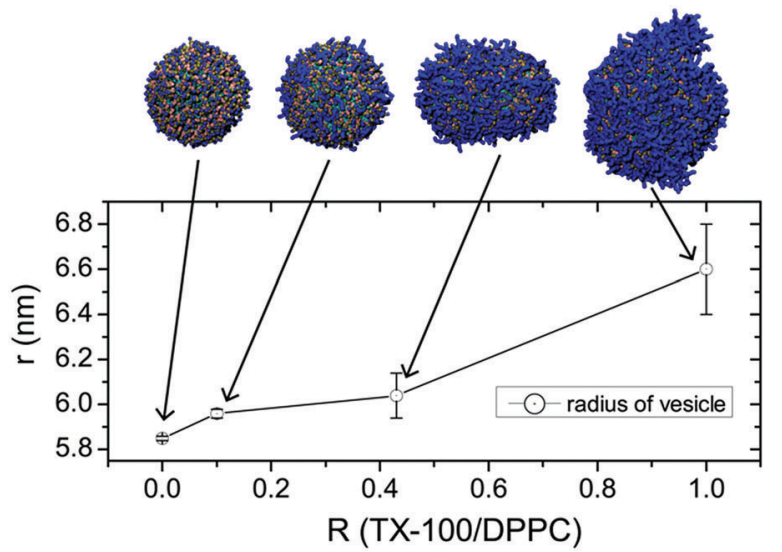

Fig. 6 Behaviour of the vesicle radius as a function of the TX-100/DPPC ratio. Snapshots showing the dimension and shape of a vesicle with the initial random TX-100 distribution at given $R$ are provided above the plot. The TX-100 molecules are shown in blue. Beads representing the polar head of DPPC molecules are shown in blue, while the aliphatic chain beads are shown in dark and light green.

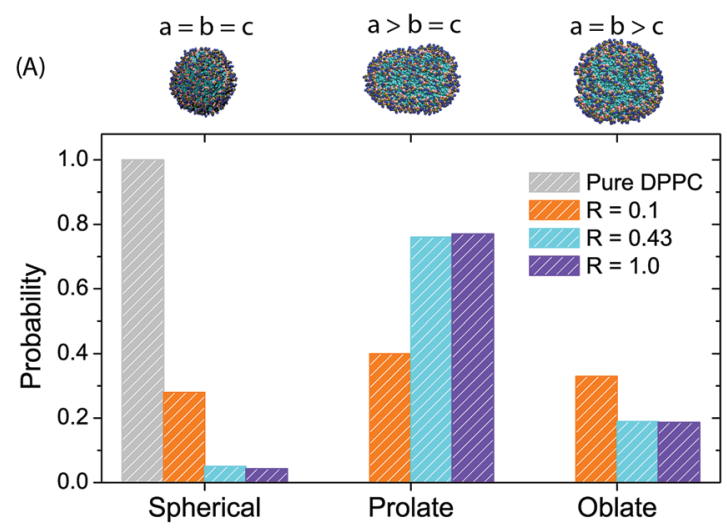

(B)
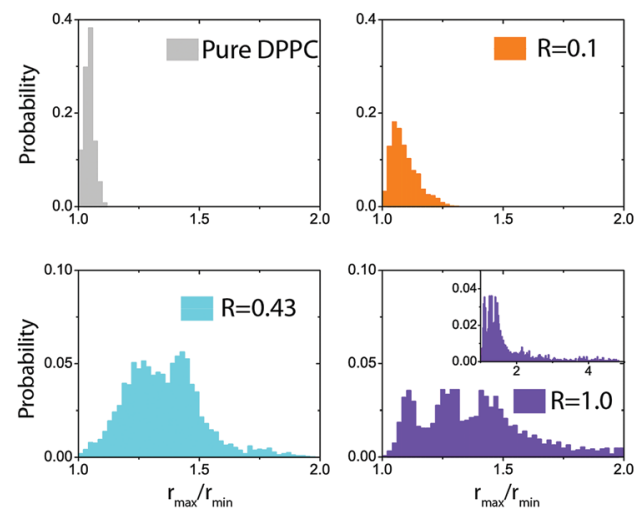

Fig. 7 (A) Probability of observing spherical, prolate or oblate vesicle shapes as a function of TX-100 concentration. On the top of the bar chart snapshots and geometrical conditions pertaining to spherical or ellipsoidal shapes are provided. (B) Distributions of asymmetry calculated as the ratio between the longest $\left(r_{\text {max }}\right)$ and shortest $\left(r_{\text {min }}\right)$ semi-axes of vesicles.

$(R=0.1)$ led to small deformations of the vesicles resulting in quasi-spherical shapes. In contrast, at a high TX-100 content, prior to disruption $(R=0.43-1.0)$, the vesicles showed strong distortion, resulting in prolate or oblate shapes. 
Consistent with its reported curvophilic nature, ${ }^{16}$ a nonuniform distribution of TX-100 was observed in non-spherical mixed vesicles. In particular, regions at a higher positive curvature, as for example surfaces of elongated vesicle or pore edges, had a higher TX-100 content.

Fig. 8 shows the statistical distribution of TX-100, calculated from the centre to the perimeter of the simulated vesicle. The TX-100 distribution was computed by slicing the vesicle with planes perpendicular to one of the axes of the micelle, and computing the average concentration of TX-100 for intervals of the corresponding elevation angle $\Delta \varphi=\left|\varphi-\varphi^{\prime}\right|$, as illustrated in the scheme of Fig. 8C. For ellipsoidal vesicles, we analysed the distribution of TX-100 along both the principal and one of
(A)

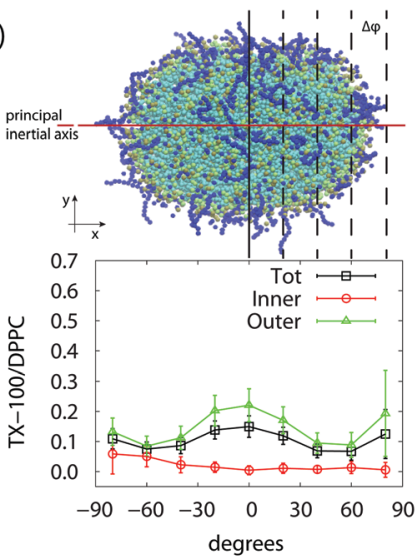

(B)

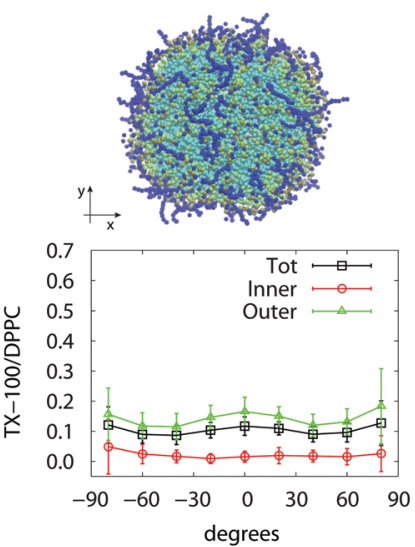

(C)

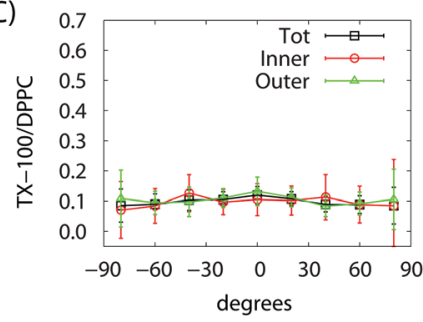

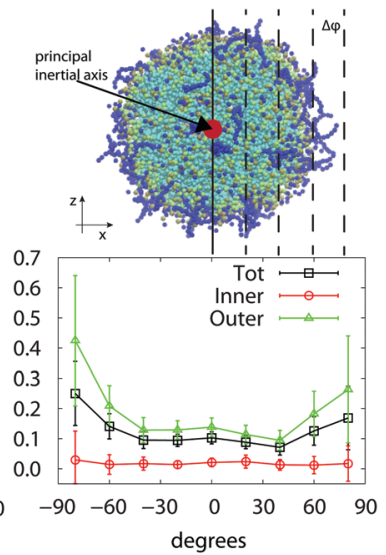
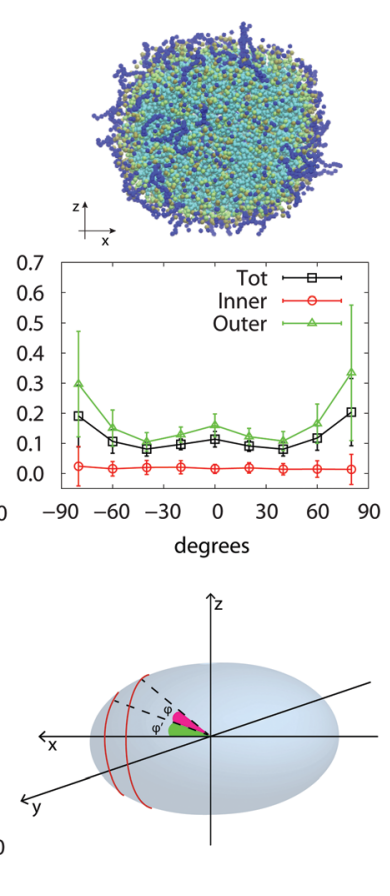

Fig. 8 TX-100 distribution inside a vesicle (with $R=0.1$ ), for two different orientations of the vesicle, as a function of shape: (A) prolate vesicle, (B) quasi-spherical shape. (C) Distribution of TX-100 in the starting spherical configuration at time $t=0.0 \mu$ s with $R=0.1$. In the same panel is reported a scheme to illustrate how the slices, on which TX-100 molecules are counted, have been selected. The TX-100 molecules are reported in dark blue. the secondary inertia axes (Fig. 8A and B). In prolate vesicles (Fig. 8A), the distribution of TX-100 in the inner layer is almost uniform in both orientations corresponding to the two axes. In contrast, the distribution in the outer layer along the principal axis of inertia shows two peaks, the first in the middle and the second corresponding to vesicle pores. Considering a different orientation of the same vesicle (right plot in Fig. 8A), the highest TX-100 content is found to be on the edge of the vesicle corresponding to the highest curvature region. A similar behaviour is also found for the quasi-spherical vesicle, but in this case the surfaces have comparable curvature radii with the result that the distributions are smoother (Fig. 8B). Nonetheless, a small difference in the curvature still corresponds to an uneven TX-100 distribution on the surface. The higher is the deviation from the ideal spherical shape, the higher is the TX-100 transmigration on positive curvature regions. Distributions calculated for vesicles at a higher TX-100 content $(R=0.43$ and 1.0), confirming such scheme, are reported in the ESI. $\dagger$

3.5.2 Mechanism of vesicle disruption. On the basis of our results and observations, we could identify the following steps facilitating the solubilization of DPPC vesicles. To this aim, we have chosen a system with a random initial TX-100 distribution in a vesicle and with a ratio $R=1.0$. From the whole simulation trajectory, we selected three different vesicle states, spherical, pored and elongated vesicles, and mixed aggregates. In Fig. 9, snapshots of each vesicle state with the corresponding TX-100 distribution plot are reported. At time $t=0 \mu \mathrm{s}$ the vesicle is perfectly spherical $\left(\boldsymbol{r}_{\max } / \boldsymbol{r}_{\min }=1.0\right)$ and the TX-100 has a uniform distribution. Initially, the vesicle starts to develop pores. This stage is accompanied by an increase in the asphericity $(t=0.16-0.8 \mu \mathrm{s})$, with TX-100 mainly distributed along the pore edge and in correspondence of the most positive curved surface areas. In addition, the difference of the TX-100 content between outer and inner layers becomes asymmetric, with a higher content in the outer layer (Fig. 9). After $\Delta t>1.0 \mu \mathrm{s}$, the cavity of the vesicle is completely lost. A complete reorganization of DPPC and TX-100 molecules occurs and a mixed aggregate is formed. The final equilibrium structure that we obtain is a bicelle-like aggregate in which the DPPC molecules form a compact, double layer raft with TX-100 molecules mainly distributed on the edges (Fig. 9). The oblate mixed micelle that we observed, with high asphericity $\left(r_{\max } / r_{\min }=4.2\right)$, is in agreement with experimental evidence from light scattering and SAXS measurements ${ }^{16,20,27}$ conducted on systems having a TX-100/lipid ratio close to $R_{\mathrm{e}}^{\mathrm{sol}}$, similar to our conditions. Snapshots showing the vesicle disruption for the other initial configurations are reported in the ESI. $\dagger$

\subsection{Vesicles with an intermediate radius of curvature}

Since the simulated vesicles are smaller when compared to the most experimentally studied systems, they can show spurious effects due to their high curvature. On the other hand, flat bilayers represent a limiting case for large vesicles, i.e. when the curvature radius goes to infinity. In this case, the TX-100 molecules will tend to increase the membrane curvature (see Scheme 2). In order to better understand the role of the vesicle 


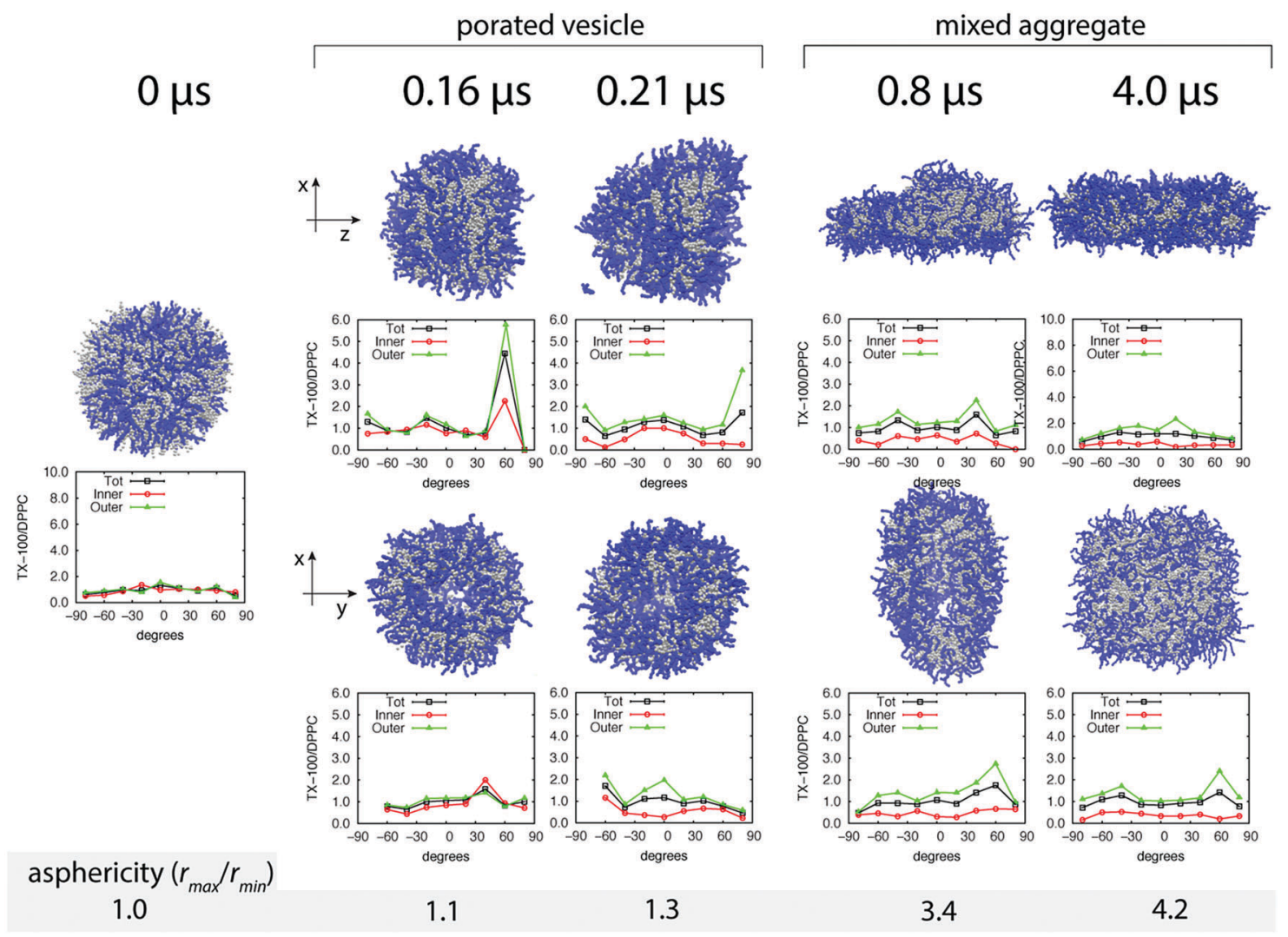

Fig. 9 TX-100 distribution as a function of vesicle shapes. The plots reported in the figure have been calculated on a system with $R=1.0$ and with a random initial TX-100 distribution. For the initial spherical vesicle, $t=0.0 \mu \mathrm{s}$, only one orientation has been reported. In the case of asphericity greater than 1.1, the plots for two representative orientations are reported. For each orientation a snapshot of the mixed vesicle is shown. To underline and make clearer the TX-100 molecule positions, they have been coloured blue, while the DPPC lipids are coloured grey.

curvature, we decided to simulate a larger vesicle with an intermediate value of the curvature radius. We selected a vesicle size that is similar to the one investigated with Dissipative Particle Dynamics (DPD), by Graftmuller and co-workers. ${ }^{95}$ They previously reported fusion of 14 and $28 \mathrm{~nm}$ diameter vesicles with a planar membrane in molecular detail, with the aim of constructing a coarse-grained model of phospholipid membranes with realistic properties, and in particular a realistic dependence of the fusion time on the membrane tension. To this aim, we performed a $4.5 \mathrm{~ns}$ simulation of a vesicle, starting from a symmetric distribution of TX-100 in the inner and outer leaflet of the bilayer, corresponding to a TX-100/DPPC ratio $R=0.43$. The starting vesicular structure, containing 5800 nonwater molecules (1745 molecules of TX-100 and 4055 molecules

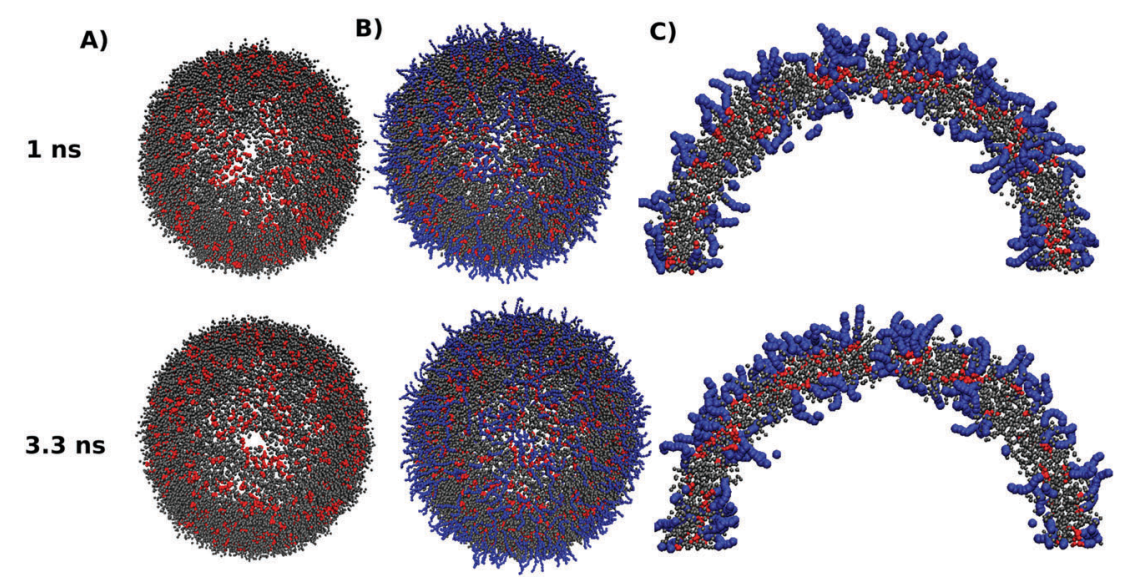

Fig. 10 Snapshots from two different views: (A) top view DPPC molecules (gray) and TX-100 hydrophobic heads (red); (B) with the TX-100 hydrophilic chains (blue); (C) side view inside the vesicle, denoting the formation of pore during the simulation time. This hole is well formed at $3.3 \mathrm{~ns}$ with TX-100. 
A)

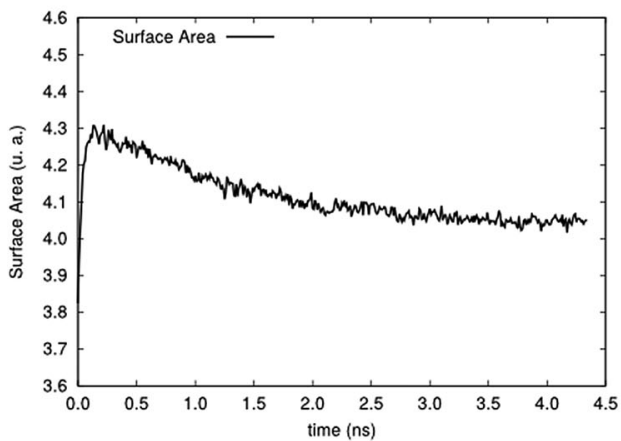

C)

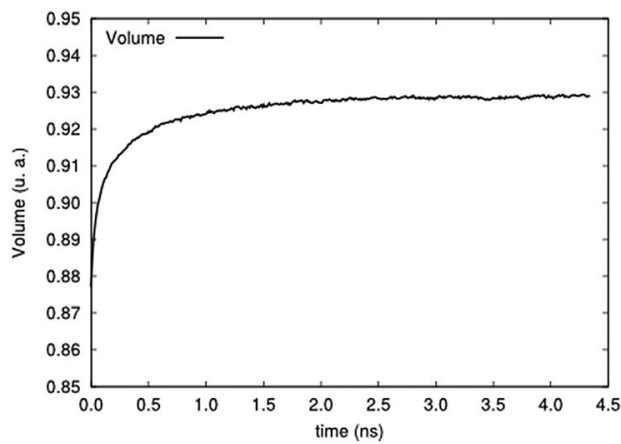

B)

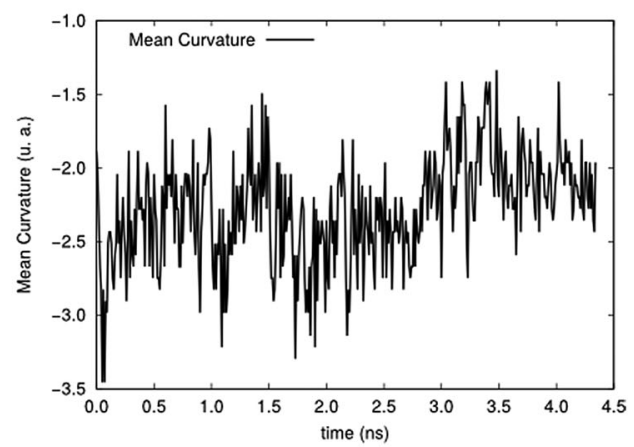

D)

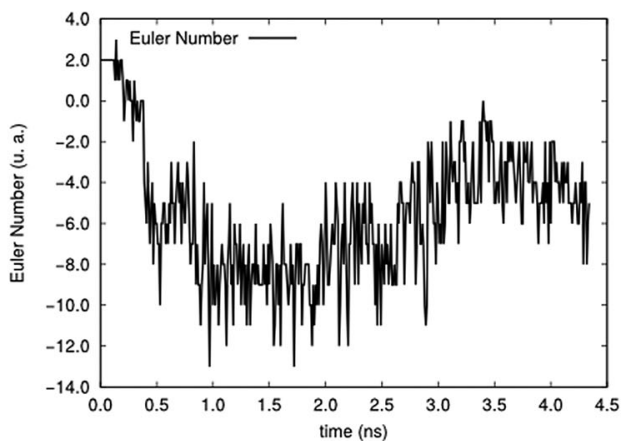

Fig. 11 Evolution of the Minkowski functionals related to (A) the surface area, (B) integral mean curvature, (C) volume and (D) Euler number for a 26 nm diameter vesicle with a TX-100/DPPC $R=0.43$ ratio, where TX-100 is symmetrically distributed.

of DPPC), quickly relaxes, by mild deflation, into a mixed TX-100/DPPC vesicle with a diameter of approximately $26 \mathrm{~nm}$ (see system XXVIII in Table S1 of the ESI $\dagger$ ). We have observed a quick deflation into a stable vesicle, as expected, followed by the formation of several small pores after only $1 \mathrm{~ns}$. The pores increase in size during a prolonged simulation, in full agreement with our findings for the smaller, more curved vesicles. In Fig. 10, snapshots of two different views of the vesicle at different times along the simulation trajectory are reported. In particular, the formation of holes is caused by TX-100 migration creating a hole contour with a high positive curvature. As can be seen in Fig. 10C, where a lateral section of the vesicle corresponding to the position of the hole is depicted, it is clear that TX-100 molecules are accumulated on the high curvature surface delimiting the pore contour.

To better quantify these findings, we have performed Minkowski Functional (MF) analysis of the solvent field ${ }^{96,97}$ (see Fig. 11). We have carefully analyzed the dependence of the MFs on the thresholding procedure, to select a proper threshold value (here $1 / 8$ of the average solvent concentration). The structure is fully quantified by four MFs: three geometrical MFs - the volume, surface area and mean curvature (made dimensionless by scaling with the volume, $40^{3} \mathrm{~nm}^{3}$, surface, $40^{2} \mathrm{~nm}^{2}$, and line length, $40 \mathrm{~nm}$, as defined by the simulation box) - and one topological property, the Euler characteristic. Especially the latter MF signals when pores are generated. From the evolution of the three geometrical MFs, we find a small monotonic decrease for the surface area (Fig. 11A) and a similarly small monotonic increase for the mean curvature (Fig. 11B) and volume (Fig. 11C). These evolutions show that the growth rate of the pores is quite small, which is consistent with the (relatively) slow molecular diffusion along the membrane that is needed to sustain this process. Instead, the Euler characteristic (Fig. 11D) shows a fast decrease from the initial value of 2 (equivalent to a full membrane without holes)

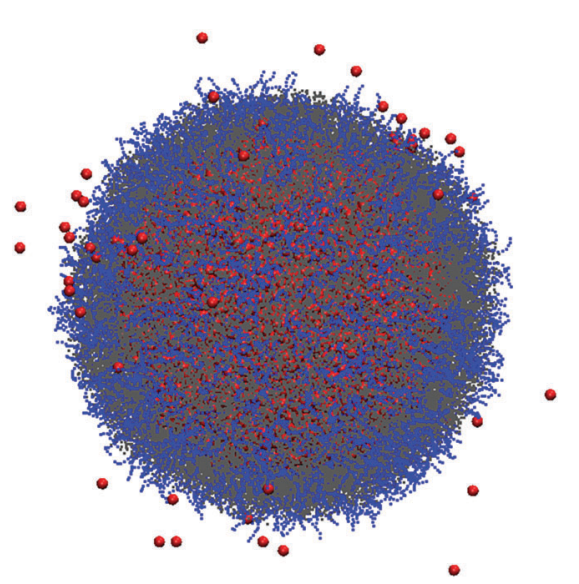

Fig. 12 Snapshot of the vesicle at $t=4.5 \mathrm{~ns}$, where the water beads (in red) present at $t=0$ inside the vesicle are reported, showing the solvent that has moved outside the vesicle. The lipid and TX-100 molecules are shown in gray and in blue, respectively. 
to slightly negative values, signalling the formation of several pores in the membrane, apparently taking place in less than 0.5 ns. Additional visual analysis (Fig. 12) indicates that the holes have to grow to a certain size before solvent can diffuse through them. In addition, the formation of several different pores is also consistent with slow molecular diffusion along the membrane, since it reflects the competition between TX-100 domain growth and pore formation. Compared to the smaller vesicle at the same concentration and its TX-100 distribution, we do not observe significant size and conformation changes on the scale of the vesicle. Pore formation is consistently observed for both vesicle sizes; for the large vesicle, the pore formation takes place earlier than for the smaller vesicle (see Fig. 4), but occurring with a smoother process.

\section{Conclusions}

A molecular-resolution computational study of the solubilization mechanism of a lipid membrane in the presence of the TX-100 detergent has been provided, for the first time to the best of our knowledge. Thanks to the computational efficiency of the MD-SCF approach, coupled with the use of CG models, we were able to systematically reach the microsecond time scales for large scale systems, and thus follow and describe the entire solubilization process of a DPPC biomembrane model.

We observed a solubilization mechanism, as a sequence of complex events, in agreement with the most accounted for three stage model. ${ }^{1}$ Our models well reproduce experiments in which the lipid membrane solubilization occurs when the effective ratio $R_{\mathrm{e}}$ (detergent/lipid) is close to the solubilization ratio $R^{\text {sol }}=1.0$. Moreover, we found different solubilization pathways, depending on fast or slow detergent partitions. Our results are in agreement with experimental evidence reported by Stuart ${ }^{42}$ and Kragh-Hansen, ${ }^{43}$ and with the generalisation to the original three stage model mechanism proposed by Lichtenberg. ${ }^{16,17,42,43}$

TX-100 is usually considered a fast solubilising detergent that can easily flip from the outer to the inner monolayer causing a rapid solubilization, ${ }^{16,28,29,31,32}$ but its surfactant function depends both on temperature ${ }^{22}$ and the lipid phase. ${ }^{3,22,29}$ When rapid solubilization does not occur, the other most probable mechanism is a slow solubilization when the bilayer becomes destabilized at a certain content of detergent that cannot easily flip into the inner layer. This causes a large increase in the curvature of the outer layer, and thus the formation of mixed micelles. ${ }^{16}$ The reasons for an unsuccessful rapid solubilization by TX-100 are typically found in the physical state of the lipid phase..$^{3,22,29}$ In fact, in the gel phase the lipids are tightly bound and do not allow the TX-100 intrusion, with a consequent absence of flips in the inner layer. In our case, DPPC molecules in both vesicles and bilayers are in a fluid state, but two different pathways of solubilization (fast for vesicles and slow for bilayers) occur. This paradox is explained on the basis of two competing processes: the flip-flop of the TX-100 and the local distortions and instabilities due to its curvophilic nature attempting to curve the flat surface. In the extreme case of a bilayer in the infinite curvature limit, the second process occurs on a lower time scale and assumes more relevance in the solubilization process. This mechanism is mentioned as a probable cause of low solubilization in ref. 16 and 17 and here it finds a confirmation, although it is an extreme case that can be studied and investigated only by means of computer simulation techniques.

An intermediate radius of curvature has also been studied, i.e. a larger $26 \mathrm{~nm}$ diameter vesicle, where TX-100 is inserted in a symmetrical way with a TX-100/DPPC ratio $R=0.43$, close to the saturation limit. In contrast to the smaller curved vesicles, the shape remains almost spherical during the simulation time. Pore formation, however, is observed for the larger vesicle and occurs earlier (less than $0.5 \mathrm{~ns}$ ) but with a smoother process.

We also proved that different MS pathways occur when the lipid surfaces have a different curvature. While strongly curved membranes allow fast flipping of detergents between the two membrane leaflets, flat membranes fall in a regime of very slow detergent partition rates. Furthermore, we reported a strong effect of the lipid surface curvature also on the TX-100 distribution. In particular, regions at a higher positive curvature, such as surfaces of elongated vesicles or pore edges, had a higher TX-100 content. At the same time, due to the curvophilic nature of TX-100, ${ }^{16}$ a higher local content of TX-100 in the membrane region promotes a surface distortion or a pore formation with the resulting membrane rupture.

The possibility of describing MS phenomena with an accurate level of resolution, also thanks to the long time scales reachable with the MD-SCF approach, paves the way to future studies on MS involving complex biological systems, including transmembrane proteins or lipid bilayers of large scale heterogeneous composition.

\section{Conflicts of interest}

There are no conflicts to declare.

\section{Acknowledgements}

G. M. thanks the HPC team of Enea (http://www.enea.it) for using the ENEA-GRID and the HPC facilities CRESCO (http:// www.cresco.enea.it) in Portici. The computing resources and the related technical support used for this work have been provided by CRESCO/ENEAGRID High Performance Computing infrastructure and its staff. ${ }^{98}$ CRESCO/ENEAGRID High Performance Computing infrastructure is funded by ENEA, the Italian National Agency for New Technologies, Energy and Sustainable Economic Development and by Italian and European research programmes, see http://www.cresco.enea.it/english for information. G. J. A. S. acknowledges funding by the VW foundation in the context of the project 'Multiscale hybrid modeling of (bio)membranes'. M. C. acknowledges the support by the Research Council of Norway (RCN) through the CoE Centre for Theoretical and Computational Chemistry (CTCC) Grant No. 179568/V30 and 171185/V30. 


\section{Notes and references}

1 A. Helenius and K. Simons, Biochim. Biophys. Acta, 1975, 415, 29-79.

2 E. A. Dennis, Arch. Biochem. Biophys., 1974, 165, 764-773.

3 E. London and D. A. Brown, Biochim. Biophys. Acta, Biomembr., 2000, 1508, 182-195.

4 J. Lasch, Biochim. Biophys. Acta, Rev. Biomembr., 1995, 1241, 269-292.

5 C. Rosano and M. Rocco, FEBS J., 2010, 277, 3190-3202.

6 M. le Maire, P. Champeil and J. V. Møller, Biochim. Biophys. Acta, Biomembr., 2000, 1508, 86-111.

7 D. A. Brown and E. London, J. Biol. Chem., 2000, 275, 17221-17224.

8 L. Hinck, I. S. Näthke, J. Papkoff and W. J. Nelson, J. Cell Biol., 1994, 125, 1327-1340.

9 I. Rabinovitz and A. M. Mercurio, J. Cell Biol., 1997, 139, 1873-1884.

10 A. Bernkop-Schnürch and G. Walker, Crit. Rev. Ther. Drug Carrier Syst., 2001, 18, 459-501.

11 D. A. Brown and E. London, Annu. Rev. Cell Dev. Biol., 1998, 14, 111-136.

12 F. Sotgia, B. Razani, G. Bonuccelli, W. Schubert, M. Battista, H. Lee, F. Capozza, A. L. Schubert, C. Minetti, J. T. Buckley and M. P. Lisanti, Mol. Cell. Biol., 2002, 22, 3905-3926.

13 S. Pfeiffer, S. D. Fuller and K. Simons, J. Cell Biol., 1985, 101, 470-476.

14 T. Hamada, R. Sugimoto, M. d. C. Vestergaard, T. Nagasaki and M. Takagi, J. Am. Chem. Soc., 2010, 132, 10528-10532.

15 A. De la Maza and J. L. Parra, Biochem. J., 1994, 303, 907-914.

16 D. Lichtenberg, H. Ahyayauch and F. M. Goñi, Biophys. J., 2013, 105, 289-299.

17 D. Lichtenberg, H. Ahyayauch, A. Alonso and F. M. Goñi, Trends Biochem. Sci., 2013, 38, 85-93.

18 D. Lichtenberg, E. Opatowski and M. M. Kozlov, Biochim. Biophys. Acta, Biomembr., 2000, 1508, 1-19.

19 D. Lichtenberg, Biochim. Biophys. Acta, Biomembr., 1985, 821, 470-478.

20 D. Lichtenberg, R. J. Robson and E. A. Dennis, Biochim. Biophys. Acta, 1983, 737, 285-304.

21 F. M. Goni, M.-A. Urbaneja, J. L. R. Arrondo, A. Alonso, A. A. Durrani and D. Chapman, Eur. J. Biochem., 1986, 160, 659-665.

22 E. Schnitzer, D. Lichtenberg and M. M. Kozlov, Chem. Phys. Lipids, 2003, 126, 55-76.

23 S. Almog, B. J. Litman, W. Wimley, J. Cohen, E. J. Wachtel, Y. Barenholz, A. Ben-Shaul and D. Lichtenberg, Biochemistry, 1990, 29, 4582-4592.

24 P. Schurtenberger, N. Mazer and W. Kaenzig, J. Phys. Chem., 1985, 89, 1042-1049.

25 H. Ahyayauch, M. I. Collado, A. Alonso and F. M. Goñi, Biophys. J., 2012, 102, 2510-2516.

26 M.-A. Urbaneja, F. M. GoÑI and A. Alonso, Eur. J. Biochem., 1988, 173, 585-588.

27 D. Lichtenberg, Y. Zilberman, P. Greenzaid and S. Zamir, Biochemistry, 1979, 18, 3517-3525.
28 T. P. Sudbrack, N. L. Archilha, R. Itri and K. A. Riske, J. Phys. Chem. B, 2011, 115, 269-277.

29 B. Mattei, A. D. C. França and K. A. Riske, Langmuir, 2015, 31, 378-386.

30 A. A. Ribeiro and E. A. Dennis, Biochim. Biophys. Acta, 1973, 232, 26-35.

31 H. Y. Fan, D. Das and H. Heerklotz, Langmuir, 2016, 32, 11655-11663.

32 H. Heerklotz, H. Szadkowska, T. Anderson and J. Seelig, J. Mol. Biol., 2003, 329, 793-799.

33 J. Sot, M. I. Collado, J. L. R. Arrondo, A. Alonso and F. M. Goñi, Langmuir, 2002, 18, 2828-2835.

34 M. A. Urbaneja, A. Alonso, J. M. Gonzalez-Mañas, F. M. Goñi, M. A. Partearroyo, M. Tribout and S. Paredes, Biochem. J., 1990, 270, 305-308.

35 H. Ahyayauch, B. Larijani, A. Alonso and F. M. Goñi, Biochim. Biophys. Acta, Biomembr., 2006, 1758, 190-196.

36 H. Heerklotz and J. Seelig, Biophys. J., 2000, 78, 2435-2440.

37 H. Heerklotz, Biophys. J., 2002, 83, 2693-2701.

38 M. Nazari, M. Kurdi and H. Heerklotz, Biophys. J., 2012, 102, 498-506.

39 S. Keller, A. Tsamaloukas and H. Heerklotz, J. Am. Chem. Soc., 2005, 127, 11469-11476.

40 Hari S. Muddana, Homer H. Chiang and Peter J. Butler, Biophys. J., 2012, 102, 489-497.

41 D. Lichtenberg, F. M. Goñi and H. Heerklotz, Trends Biochem. Sci., 2005, 30, 430-436.

42 M. C. A. Stuart and E. J. Boekema, Biochim. Biophys. Acta, Biomembr., 2007, 1768, 2681-2689.

43 U. Kragh-Hansen, M. le Maire and J. V. Møller, Biophys. J., 1998, 75, 2932-2946.

44 H. Ahyayauch, M. Bennouna, A. Alonso and F. M. Goñi, Langmuir, 2010, 26, 7307-7313.

45 M. M. A. Elsayed and G. Cevc, Biochim. Biophys. Acta, Biomembr., 2011, 1808, 140-153.

46 M. Almgren, Biochim. Biophys. Acta, Biomembr., 2000, 1508, 146-163.

47 K. Edwards and M. Almgren, J. Colloid Interface Sci., 1991, 147, 1-21.

48 E. London and G. W. Feigenson, J. Lipid Res., 1979, 20, 408-412.

49 H. Ahyayauch, M. I. Collado, F. M. Goñi and D. Lichtenberg, FEBS Lett., 2009, 583, 2859-2864.

50 S. Keller, H. Heerklotz, N. Jahnke and A. Blume, Biophys. J., 2006, 90, 4509-4521.

51 C. Arnulphi, J. Sot, M. García-Pacios, J.-L. R. Arrondo, A. Alonso and F. M. Goñi, Biophys. J., 2007, 93, 3504-3514.

52 D. Levy, A. Gulik, M. Seigneuret and J. L. Rigaud, Biochemistry, 1990, 29, 9480-9488.

53 A. Prado, J. L. R. Arrondo, A. Villena, F. M. Goñi and J. M. Macarulla, Biochim. Biophys. Acta, Biomembr., 1983, 733, 163-171.

54 S. J. Marrink and A. E. Mark, J. Am. Chem. Soc., 2003, 125, 11144-11145.

55 V. Knecht, M. Müller, M. Bonn, S.-J. Marrink and A. E. Mark, J. Chem. Phys., 2005, 122, 024704. 
56 K. A. Scott, P. J. Bond, A. Ivetac, A. P. Chetwynd, S. Khalid and M. S. P. Sansom, Structure, 2008, 16, 621-630.

57 P. J. Bond, J. Holyoake, A. Ivetac, S. Khalid and M. S. P. Sansom, J. Struct. Biol., 2007, 157, 593-605.

58 A. A. Gurtovenko and I. Vattulainen, J. Phys. Chem. B, 2007, 111, 13554-13559.

59 W. F. D. Bennett, J. L. MacCallum, M. J. Hinner, S. J. Marrink and D. P. Tieleman, J. Am. Chem. Soc., 2009, 131, 12714-12720.

60 H. J. Risselada and S. J. Marrink, Phys. Chem. Chem. Phys., 2009, 11, 2056-2067.

61 C. A. López, A. H. de Vries and S. J. Marrink, PLoS Comput. Biol., 2011, 7, e1002020.

62 M. Simunovic, C. Mim, T. C. Marlovits, G. Resch, V. M. Unger and G. A. Voth, Biophys. J., 2013, 105, 711-719.

63 G. Milano and T. Kawakatsu, J. Chem. Phys., 2009, 130, 214106.

64 S. Izvekov and G. A. Voth, J. Phys. Chem. B, 2005, 109, 2469-2473.

65 S. Bandyopadhyay, J. C. Shelley and M. L. Klein, J. Phys. Chem. B, 2001, 105, 5979-5986.

66 H. J. Risselada and S. J. Marrink, Proc. Natl. Acad. Sci. U. S. A., 2008, 105, 17367-17372.

67 S. J. Marrink, H. J. Risselada, S. Yefimov, D. P. Tieleman and A. H. de Vries, J. Phys. Chem. B, 2007, 111, 7812-7824.

68 S.-J. Marrink and A. E. Mark, Biophys. J., 2004, 87, 3894-3900.

69 S. J. Marrink, A. H. de Vries and A. E. Mark, J. Phys. Chem. B, 2004, 108, 750-760.

70 S. Izvekov and G. A. Voth, J. Chem. Theory Comput., 2006, 2, 637-648.

71 J. C. Shelley, M. Y. Shelley, R. C. Reeder, S. Bandyopadhyay, P. B. Moore and M. L. Klein, J. Phys. Chem. B, 2001, 105, 9785-9792.

72 G. Milano, T. Kawakatsu and A. De Nicola, Phys. Biol., 2013, 10, 045007.

73 A. De Nicola, Y. Zhao, T. Kawakatsu, D. Roccatano and G. Milano, J. Chem. Theory Comput., 2011, 7, 2947-2962.

74 A. Pizzirusso, A. De Nicola and G. Milano, J. Phys. Chem. B, 2016, 120, 3821-3832.

75 A. De Nicola, T. Kawakatsu, C. Rosano, M. Celino, M. Rocco and G. Milano, J. Chem. Theory Comput., 2015, 11, 4959-4971.

76 W. Shinoda, R. DeVane and M. L. Klein, Soft Matter, 2011, 7, 6178-6186.

77 A. V. Sangwai and R. Sureshkumar, Langmuir, 2011, 27, 6628-6638.

78 W. Shinoda, R. DeVane and M. L. Klein, Soft Matter, 2008, 4, 2454-2462.
79 R. D. Groot and K. L. Rabone, Biophys. J., 2001, 81, 725-736. 80 C.-M. Lin, G.-P. Chang, H.-K. Tsao and Y.-J. Sheng, J. Chem. Phys., 2011, 135, 045102.

81 L. Monticelli, S. K. Kandasamy, X. Periole, R. G. Larson, D. P. Tieleman and S.-J. Marrink, J. Chem. Theory Comput., 2008, 4, 819-834.

82 G. Milano and T. Kawakatsu, J. Chem. Phys., 2010, 133, 214102.

83 A. De Nicola, Y. Zhao, T. Kawakatsu, D. Roccatano and G. Milano, Theor. Chem. Acc., 2012, 131, 1-16.

84 A. De Nicola, T. Kawakatsu and G. Milano, Macromol. Chem. Phys., 2013, 214, 1940-1950.

85 A. De Nicola, S. Hezaveh, Y. Zhao, T. Kawakatsu, D. Roccatano and G. Milano, Phys. Chem. Chem. Phys., 2014, 16, 5093-5105.

86 T. A. Soares, S. Vanni, G. Milano and M. Cascella, J. Phys. Chem. Lett., 2017, 8, 3586-3594.

87 Y. Zhao, A. De Nicola, T. Kawakatsu and G. Milano, J. Comput. Chem., 2012, 33, 868-880.

88 S. Pronk, S. Páll, R. Schulz, P. Larsson, P. Bjelkmar, R. Apostolov, M. R. Shirts, J. C. Smith, P. M. Kasson, D. van der Spoel, B. Hess and E. Lindahl, Bioinformatics, 2013, 184, 2856-2859.

89 H. J. C. Berendsen, J. P. M. Postma, W. F. van Gunsteren, A. DiNola and J. R. Haak, J. Chem. Phys., 1984, 81, 3684-3690.

90 H. C. Andersen, J. Chem. Phys., 1980, 72, 2384-2393.

91 P. Fromherz, C. Rocker and D. Ruppel, Faraday Discuss. Chem. Soc., 1986, 81, 39-48.

92 A. Alonso, R. Sáez, A. Villena and F. Goñi, J. Membr. Biol., 1982, 67, 55-62.

93 A. Alonso, A. Villena and F. M. Goñi, FEBS Lett., 1981, 123, 200-204.

94 K. Edwards, M. Almgren, J. Bellare and W. Brown, Langmuir, 1989, 5, 473-478.

95 A. Grafmüller, J. Shillcock and R. Lipowsky, Biophys. J., 2009, 96, 2658-2675.

96 G. J. A. Sevink and A. V. Zvelindovsky, J. Chem. Phys., 2004, 121, 3864-3873.

97 K. Michielsen and H. De Raedt, Phys. Rep., 2001, 347, 461-538.

98 G. Ponti, F. Palombi, D. Abate, F. Ambrosino, G. Aprea, T. Bastianelli, F. Beone, R. Bertini, G. Bracco, M. Caporicci, B. Calosso, M. Chinnici, A. Colavincenzo, A. Cucurullo, P. Dangelo, M. D. Rosa, P. D. Michele, A. Funel, G. Furini, D. Giammattei, S. Giusepponi, R. Guadagni, G. Guarnieri, A. Italiano, S. Magagnino, A. Mariano, G. Mencuccini, C. Mercuri, S. Migliori, P. Ornelli, S. Pecoraro, A. Perozziello, S. Pierattini, S. Podda, F. Poggi, A. Quintiliani, A. Rocchi, C. Sciò, F. Simoni and A. Vita, 2014 International Conference on High Performance Computing \& Simulation (HPCS), 2014. 